\title{
New statistical goodness of fit techniques in noisy inhomogeneous inverse problems
}

\section{With application to the recovering of the luminosity distribution of the Milky Way}

\author{
N. Bissantz ${ }^{1}$ and A. Munk ${ }^{2}$ \\ 1 Astronomisches Institut der Universität Basel, Venusstr. 7, 4102 Binningen/Basel, Switzerland \\ 2 Fakultät für Mathematik und Informatik der Universität GH Paderborn, Warburgerstr. 100, \\ 33098 Paderborn, Germany
}

Received 16 June 2000 / Accepted 12 June 2001

\begin{abstract}
The assumption that a parametric class of functions fits the data structure sufficiently well is common in fitting curves and surfaces to regression data. One then derives a parameter estimate resulting from a least squares fit, say, and in a second step various kinds of $\chi^{2}$ goodness of fit measures, to assess whether the deviation between data and estimated surface is due to random noise and not to systematic departures from the model. In this paper we show that commonly-used $\chi^{2}$-measures are invalid in regression models, particularly when inhomogeneous noise is present. Instead we present a bootstrap algorithm which is applicable in problems described by noisy versions of Fredholm integral equations of the first kind. We apply the suggested method to the problem of recovering the luminosity density in the Milky Way from data of the DIRBE experiment on board the COBE satellite.
\end{abstract}

Key words. methods: data analysis - methods: statistical - Galaxy: structure

\section{Introduction}

Regression problems arise in almost any branch of physics, including astronomy and astrophysics. In general, the problem of estimating a regression function (or surface) occurs when a functional relationship between several quantities of interest has to be found from blurred observations $\left(y_{i}, t_{i}\right), i=1, \cdots, n$. Here $y=\left(y_{1}, \cdots, y_{n}\right)$ denotes a vector of measurements (response vector) and $t=\left(t_{1}, \cdots, t_{n}\right)$ a quantity which affects the response vector in a systematic but blurred way, which is to be investigated. This systematic component is usually denoted as the regression function $E\left[Y_{i}\right]=\omega\left(t_{i}\right)$. Note that $Y_{i}$ is a random variable, of which $y_{i}$ is a realisation. If $t_{i} \in \mathbb{R}$, this includes signal detection problems or image restoration if $t_{i} \in \mathbb{R}^{2}$. Many problems bear the additional difficulty that the quantity of interest is not directly accesible to the observations $y$ and the relationship has to be expressed by a noisy version of a Fredholm integral equation of the first kind, viz.

$y_{i}=\omega\left(t_{i}\right)+\varepsilon_{i}=(\mathbf{K} \rho)\left(t_{i}\right)+\varepsilon_{i}$,

Send offprint requests to: N. Bissantz, e-mail: bissantz@astro.unibas.ch where $\mathbf{K}$ is a given integral operator, $\rho$ the regression function to be reconstructed and $\varepsilon=\left(\varepsilon_{1}, \cdots, \varepsilon_{n}\right)$ a vector of independent random quantities (error), due to imprecise measurements and other sources of noise. More precisely, we assume that the expectation of $y_{i}$ is given by $(\mathbf{K} \rho)\left(t_{i}\right)$ and inhomogeneous noise might be present, i.e. the variance $\sigma_{i}^{2}$ of the noise $\varepsilon_{i}$ (and possibly higher moments, too) depends on the grid point $t_{i}$. There is a vast amount of literature concerning statistical theory for the estimation of $\rho$, we mention only Wand \& Jones (1995) for direct regression and Nychka \& Cox (1989) or van Rooij \& Rymgaart (1996) for the inverse (sometimes denoted as indirect) case, as in Eq. (1). (Inverse) regression models capture various examples from astronomy and physics (cf. Bertero 1989 or Lucy 1994a, 1994b for an overview). Such an example is the reconstruction of the three-dimensional luminosity in the Milky Way [MW], which will be discussed extensively in Sect. 5. In this example, $\rho$ will be a three-dimensional density of the MW, $\mathbf{K}$ the operator that projects this density to the sky, $\mathbf{K} \rho\left(t_{i}\right)$ the resulting surface brightness at the sky position $t_{i}=(l, b)_{i}$ and $y_{i}$ the observed surface brightness at $(l, b)_{i}$.

Reconstruction procedures (estimation) of $\rho$ in general depend on various a priori assumptions about $\rho$, such as smoothness properties or geometrical constraints, e.g. 
monotonicity. The most common assumptions are that $\rho$ has a particular structure and shape, depending on some unknown parameter $\vartheta$. Such an assumption is denoted as a parametric model. Typically, these structural assumptions arise from physical reasoning and approximation procedures. Often, however, it is not completely clear whether these assumptions are satisfied and therefore it is an important task to investigate empirically (by means of the data at hand) whether the resulting model is valid. Therefore, in this paper we discuss recent methodology for the investigation of the adequacy of such a parametric model $U=\left\{\rho_{\vartheta}\right\}_{\vartheta \in \Theta}, \Theta \subset \mathbb{R}^{d}$. This will be done for regular regression problems as well as for the inverse case, as in $(1)$.

The paper is organized as follows. In the next section we briefly review common practices to judge the goodness of fit of a model $U$. It is shown that classical goodness of fit approaches, such as least square statistics are insufficient from many methodological points of view, particulary when inhomogeneous noise is present, i.e. the variation $\sigma_{i}^{2}$ of the error $\varepsilon_{i}$ is expected to vary with the grid point (covariate) $t_{i}$. We show in Sect. 2 that statistically valid conclusions about the goodness of fit from the residuals $\sum r_{i}^{2}=\sum\left(y_{i}-\left(\mathbf{K} \rho_{\hat{\theta}}\right)\left(t_{i}\right)\right)^{2}$ (or variants of it) are impossible in general, particularly when inhomogeneous noise is present, as is the case in our data example. This is mainly due to the fact that in the inhomogeneous case the distribution of $\sum r_{i}^{2}$ depends on the whole vector $\left(\sigma_{1}^{2}, \ldots, \sigma_{n}^{2}\right)$ which is in general unknown. Therefore, we suggest in Sect. 3 a measure of fit which is based on "smoothed residuals" and which allows for the calculation of the corresponding probability distribution. In Sect. 4, a bootstrap resampling algorithm is suggested which allows the algorithmic reconstruction of the distribution of the suggested goodness of fit quantity. The use of bootstrap techniques is well documented in astronomy (cf. Barrow et al. 1984; Simpson \& Mayer 1986; van den Bergh \& Morbey 1984 for various applications). The work similar in spirit to ours is Bi \& Börner's (1994) residual type bootstrap, used as a method for nonparametric estimation in inverse problems. As a byproduct we show, however, that this residual bootstrap is insufficient in the case of inhomogeneous noise in the data and a so-called "wild" bootstrap has to be used instead.

Finally we will apply our new method in Sect. 5 to the fit of the COBE/DIRBE $L$-band data. We use a functional form for a parametric model of the MW as presented by Binney et al. (1997, hereafter BGS) and find similar structural parameters of the Milky disk and bulge, except for the scale height of the disk which we find to be about $25 \%$ smaller.

\section{Common $\chi^{2}$ methods of judging the quality of fit}

One of the most popular techniques for finding a proper fit of a given model $U$ to a given set of data $y_{1}, \cdots, y_{n}$ is to minimize a (penalized) weighted sum of squares

$Q_{\tilde{w}}^{n}(\vartheta):=\sum_{i=1}^{n} \tilde{w}_{i}\left(y_{i}-\omega_{\vartheta}\left(t_{i}\right)\right)^{2}$

where the $\tilde{w}_{i}$ denotes some weighting scheme and the model is $\omega_{\vartheta}\left(t_{i}\right)=\left(\mathbf{K} \rho_{\vartheta}\right)\left(t_{i}\right)$. This leads to a weighted least squares estimator (WLSE) of the optimal model parameter, $\hat{\vartheta}_{\tilde{w}}$. However, it is well known that a proper choice of the weights $\tilde{w}_{1}, \ldots, \tilde{w}_{n}$ depends on the (possibly position-dependent) random noise in the data. For example, under an uncorrelated normal error assumption, if the variance $\sigma_{i}^{2}$ of the error $\varepsilon_{i}$ is assumed to be known, a suitable choice of weights is $w_{i}=\sigma_{i}^{-2}$ in order to take into account the local variability of the observations at the grid point $t_{i}$. Particularly, in this case, the ordinary, unweighted least squares estimator is known to be insufficient (Gallant 1987), because the log likelihood of the model is proportional to $Q_{\tilde{w}}^{n}(\vartheta)$. Only if the variance pattern is homogeneous (i.e. $\sigma_{i}^{2}=\sigma^{2}$ ) are unweigthed least squares estimators optimal. The weighted least squares approach is, however, limited if the local variances $\sigma_{i}^{2}$ in the data points are unknown. The $\sigma_{i}^{2}$ then have to be estimated from the data. This is often neglected. It is also common practice to consider $Q_{w}^{n}$ in order to judge the quality of fit achieved by the regression function (where the weights $w_{i}$ may sometimes be different to those used in computing the WLSE). Here, a "large" value of $Q_{w}^{n}$ is used as an indicator for a "significant" deviation between the observations and the model to be fitted. We will investigate this in more detail in what follows, and emphasize the case of nonhomogeneous variances.

In general, the most important properties required of any goodness of fit (GoF) quantity $\chi^{2}$ such as $\chi_{w}^{2}$ are that

1) we are able to detect with high probability deviations from the model we have in mind (often denoted by statisticians as good "power");

2 ) we can quantify the probability that $\chi^{2}$ exceeds some "critical value" in order to obtain a precise probabilistic analysis (computing significance levels, confidence intervals, etc.).

As a rough rule of thumb often

$\frac{1}{n-d} \chi_{w}^{2} \approx 1$

is taken as a measure of evidence for the model $U$ and hence for the fit of $\omega_{\hat{\vartheta}_{\tilde{w}}}$. Here $d$ denotes the dimension (number of parameters) of $U$ and $n$ the number of data points. Examples of the use of this kind of statistics can be found in Alcock et al. (1997) and in Dwek et al. (1995) in the context of discriminating between several models. A related well-known quantity is the sum of squares of "expected minus observed divided by expected" for testing distributional assumptions, such as normality of the data (cf. Cox \& Hinkley 1974). Bi \& Börner (1994) considered a similar quantity in a deconvolution setup which is, using 
the notation of (1)

$\sum_{i=1}^{n} \frac{\hat{r}_{i}^{2}}{\omega_{\hat{\vartheta}}\left(t_{i}\right)}$

This obviously downweights the influence of residuals if the corresponding predicted value $\omega_{\hat{\vartheta}}\left(t_{i}\right)$ is large. Another option is to consider the absolute deviation of the predicted and observed values, which leads to a more robust version of $\chi^{2}$, or even more general distance measures can be used (Cook \& Weisberg 1999; Hocking 1996; Lucy 1994a; 1994b). In the following we will argue that an approach like $\chi_{w}^{2}$ is not valid in regression models such as (1), particularly when the noise is inhomogeneous or the residuals are not gaussian. To this end we briefly discuss the (asymptotic) distribution of the abovementioned quantities.

In order to get a first insight into the probabilistic behaviour of statistics such as $\chi^{2}$, used as a quantitative measure of fit, it is helpful to consider the distribution in the simplest case when $\omega \equiv 0$. A simple calculation then shows that (assuming a normal distribution of the data) $\chi^{2}=\sum_{i=1}^{n} y_{i}^{2}$ is distributed as a sum of normally distributed variables having the expectation $E\left[\chi^{2}\right]=\sum_{i=1}^{n} \sigma_{i}^{2}$, and variance $V\left[\chi^{2}\right]=2 \sum_{i=1}^{n} \sigma_{i}^{4}$. Hence, already in this simple case it can be seen that the determination of the law of $\chi^{2}$ is practically impossible if the variances $\sigma_{i}^{2}$ are not known. Then it is difficult to quantify what a "too large value of $\chi 2$ " means, because this will depend on the unknown quantities $\sigma_{1}^{2}, \ldots, \sigma_{n}^{2}$, and a rule as in (2) can lead in principle to any result in favour or against the model $\omega \equiv 0$. We mention that standardisation by the predicted values as in (3) does not avoid this problem. This is in contrast to goodness of fit problems for the assessment of distribution assumptions, i.e. when one investigates by a $\chi^{2}$ measure whether a population is normal, say (Cox \& Hinkley 1974). Note, that the case of homoscedastic regression models (i.e. the distribution of the noise is identical for all data points) is somewhat simpler, because here the expectation $E\left[\chi^{2}\right]=n \sigma^{2}$ and the square root of the variance $V\left[\chi^{2}\right]=2 n \sigma^{4}$ is proportional, i.e. the signal to noise ratio

$$
\frac{E\left[\chi^{2}\right]}{\sqrt{V\left[\chi^{2}\right]}}=\sqrt{n / 2}
$$

only depends on the number of data points $n$. Here, a model-free estimator of $\sigma^{2}$ can be used as a reference scale (Hart 1997).

Many attempts were made in order to find simple approximations of the distribution for $\chi_{w}^{2}$. Among them a quite attractive option is use of a bootstrap method, an algorithmic approximation of the true law (see Efron \& Tibshirani 1993 for an overview and many applications). Bootstrapping random quadratic forms (such as $\left.\chi^{2}\right)$ is, however, a rather delicate matter, because standard bootstrap algorithms such as Efron's (1979) $n$ out of $n$ bootstrap are inconsistent (Babu \& Shankya 1984;
Shao \& Tu 1995), i.e. the distribution is not approximated correctly with increasing number of observations.

The use of a particular bootstrap algorithm was indeed suggested by Bi \& Börner (1994) in the context of assessing the goodness of fit in deconvolution models. We mention that their bootstrap algorithm, however, is asymptotically not correct in inhomogeneous models. Interestingly, the suggested algorithm is similar in spirit to the so called residual bootstrap (i.e. drawing random samples with replacement from the residuals $r_{i}$ ) which is well documented in the statistical literature (cf. Davison \& Hinkley 1997, p. 281) for the estimation of the regression parameters).

Despite the abovementioned difficulties, the main problem encountered with the naive use of $\chi^{2}$ in regression models as a measure of GoF is that asymptotically (here and in the following, asymptotically means the sample size tends to infinity) the law of $\chi^{2}$ does in general not converge asymptotically to any reasonable quantity, in contrast to goodness of fit testing for distributional assumptions. Even after rescaling by $1 / \sqrt{n}$ in order to force the variance

$$
V\left[1 / \sqrt{n} \chi^{2}\right]=2 / n \sum_{i=1}^{n} \sigma_{i}^{4} \stackrel{n \rightarrow \infty}{\longrightarrow} 2 \int \sigma^{4}(t) H(\mathrm{~d} t)
$$

to converge (here it is assumed that the scheme of grid points can be described asymptotically by a distribution $H$ (Dette \& Munk 1998)) gives

$$
E\left[1 / \sqrt{n} \chi^{2}\right]=\sqrt{n}\left(1 / n \sum_{i=1}^{n} \sigma_{i}^{2}\right)=O(\sqrt{n}) \rightarrow \infty
$$

which shows that $1 / \sqrt{n} \chi^{2}$ does not converge to any reasonable quantity. Note that if we use $\chi^{2} / n$ the variance tends to zero. Also observe that subtracting $E\left[1 / \sqrt{n} \chi^{2}\right]$ from $\chi^{2}$ will not provide a way out of the dilemma because this value depends on the (unknown) variances $\sigma_{i}^{2}$.

In summary, we see that without explicit knowledge of the variances $\sigma_{i}^{2}$, the use of $\chi^{2}$ as a quantitative measure of validity of a model is not appropriate.

Due to the above-described difficulties, statisticians throughout the last two decades have extensively studied the problem of checking the goodness of fit in regression models. It is beyond the scope of this paper to review this work; many references can be found in the recent monograph by Hart (1997). Among the variety of procedures suggested so far, we mention methods which are based on model selection criteria, such as Akaike's (1974) information criterion (Eubank \& Hart 1992; Aerts et al. 1999) and methods which compare nonparametric estimators with a parametric estimator. To this end Azzalini \& Bowman (1993), Härdle \& Mammen (1993) and Müller (1992) used a kernel estimator, Cox et al. (1988) smoothing splines and Mohdeb \& Mokkadem (1998) a Fourier series estimator. However, the applicability of many of these methods is often limited. For example, Härdle \& Mammen's test is confronted with bias problems, whereas other procedures 
are only applicable for homogeneous errors or when the error distribution is completely known (Eubank \& Hart 1992; Aerts et al. 1999). Another serious difficulty arises with the nonparametric estimation of the signal as the dimension $k$ of the grid points increases. This is sometimes denoted as the curse of dimensionality (Wand \& Jones 1995; Bowmann \& Azzalini 1997). A rough rule of thumb is that the number of observations required in dimension $k$ is $n^{k}$ in order to obtain the same precision of the estimate of $\omega$. Hence, the precision induced by 100 observations on the real line is approximately the same as 10000 drawn from the plane. Furthermore, measurements often cannot be taken equidistantly over a grid, which leads to sparse data structures causing further difficulties with increasing dimension. One should also note that another difficulty consists of transferring these methods to the case of inverse problems, a situation which up to now has never been treated.

\section{A new method}

Munk \& Ruymgaart (1999) have developed a general regression methodology which remains valid in the heteroscedastic case (i.e. the distribution of the noise depends on the data point) with arbitrary dimensions of the grid points. The underlying idea dates back to H. Cramér and can be summarized as "smoothing the residuals" in order to obtain asymptotical stabilization of the test criterion. In our context this reads as follows. Let $\mathbf{T}$ denote an injective smoothing linear integral operator with associated integral kernel $T(\cdot, \cdot)$, i.e.

$\mathbf{T}[f](u)=\int T(u, t) f(t) \mathrm{d} t$.

Note that since $\mathbf{T}$ is an integral operator, $\mathbf{T}[f]$ is again a function. Now consider the transformed distance between the parametric model $V=\left\{\omega_{\vartheta}\right\}_{\vartheta \in \Theta}$ and the distribution $\omega$, which underlies the observations $y_{i}=\omega\left(t_{i}\right)+\varepsilon_{i}$ (cf. Sect. 1),

$D^{2}(g)=D^{2}(\mathbf{T}[\omega])=\min _{\vartheta \in \Theta}\left\|\mathbf{T}\left[\omega-\omega_{\vartheta}\right]\right\|^{2}$

where $g=\mathbf{T}[\omega]$ denotes the smoothed version of $\omega$ and the norm \|. $\|$ refers to some $L^{2}$-norm to be specified later on. The smoothed distance $D^{2}$ serves now as a new measure of goodness of fit and has to be estimated from data. This will be done by numerical minimization of the empirical counterpart of the r.h.s. of $(5),\left\|\hat{g}-g_{\vartheta}\right\|^{2}=\chi_{D}^{2}(\vartheta)$ where $\hat{g}(u)=1 / n \sum_{i=1}^{n} y_{i} T\left(u, t_{i}\right)$ denotes an estimator of $g(u)$. In addition this provides us with a smoothed estimator $\hat{\vartheta}_{\mathbf{T}}$ of the value $\vartheta_{\mathbf{T}}^{*}$ for which the minimum in (5) is achieved. In Munk \& Ruymgaart (1999) the kernel

$T(u, t)=\min (u, t)$,

was suggested (see Appendix A1), which will also be used in the following, and which amounts to a cumulative smoothing. Not that for $k$-dimensional $u$ and $t$ the minimum has to be understood componentwise as

$\min (u, t)=\prod_{i}^{k} \min \left(u_{i}, t_{i}\right)$

We mention that other choices of $T$ are possible (cf. Sect. A).

The reasoning behind this approach is that direct estimation of $\omega$ is a rather difficult task, whereas estimation of the smoothed transformation $g=\mathbf{T}(\omega)$ is much simpler. Furthermore, the distribution of the minimizer of $\chi_{D}^{2}(\vartheta)$ becomes tractable. If we denote the minimum of $\left\|\hat{g}-g_{\vartheta}\right\|^{2}$ as $\hat{D}^{2}$ one can show under very mild regularity conditions that (Munk \& Ruymgaart 1999) the distribution of $n \hat{D}^{2}$ converges to

$\sum_{i=1}^{\infty} \lambda_{i} \chi_{i}^{2}$

where the $\lambda_{i}$ denotes a decreasing sequence of positive numbers which depend on the best model parameter $\vartheta_{\mathbf{T}}^{*}$, which is the minimizing $\vartheta$ in (5), the model space $U$ and the unknown distribution of the error $\varepsilon$, including the variance function $\sigma^{2}\left(t_{i}\right)$. This makes a direct application of this limit law difficult and hence a peculiar bootstrap algorithm is suggested in the following which can be shown to be asymptotically consistent, i.e. the asymptotic limit law of this algorithm is the same as in (6). The following idea of the so called "wild bootstrap" dates back to Wu (1986) and was applied by Stute et al. (1998) to a testing problem similar to the one above.

\section{The wild bootstrap algorithm}

The true distribution (6) of $\hat{D}^{2}$ depends on the unknown $\lambda_{i}$. It is therefore not possible to use this distribution for practical purposes. However, it is possible to approximate the distribution numerically using the following bootstrap algorithm:

Step 1: (Generate residuals). Compute residuals

$\hat{\varepsilon}_{i}:=y_{i}-\omega_{\hat{\vartheta}_{\mathbf{T}}}\left(t_{i}\right), \quad i=1, \cdots, n$

where $\hat{\vartheta}_{\mathbf{T}}$ denotes a solution of the minimization of

$\hat{D}^{2}:=\chi^{2}\left(\hat{\vartheta}_{\mathbf{T}}\right):=\min _{\vartheta \in \Theta}\left\|\hat{g}-\mathbf{T} \omega_{\vartheta}\right\|^{2}$.

Step 2: (The "wild" part). Generate new random variables $c_{i}^{*}, i=1, \ldots, n$, which do not depend on the data, where each $c_{i}^{*}$ is distributed to a distribution which assigns probability $(\sqrt{5}+1) / 2 \sqrt{5}$ to the value $(-\sqrt{5}-1) / 2$ and $(\sqrt{5}-1) / 2 \sqrt{5}$ to the value $(\sqrt{5}+1) / 2$. See Fig. 1 for a visualization of this probability distribution.

Step 3: (Bootstrapping residuals). Compute $\varepsilon_{i}^{*}:=\hat{\varepsilon}_{i} c_{i}^{*}$ and $y_{i}^{*}=\omega_{\hat{\vartheta}_{\mathbf{T}}}+\varepsilon_{i}^{*}$. This gives a new data vector $\left(y_{i}^{*}, t_{i}\right)_{i=1, \ldots, n}$. 


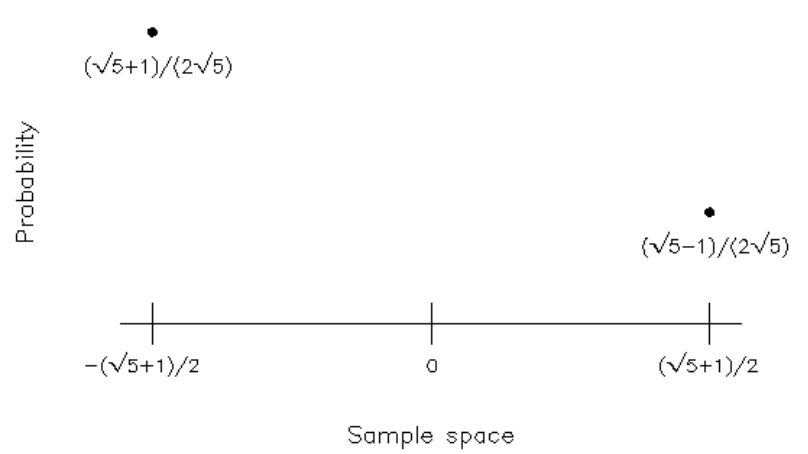

Fig. 1. Binary probability distribution required in step 2 of the wild bootstrap algorithm.

Step 4: (Compute the target). Compute $\hat{D}^{2, *}$ with $\left(y_{i}^{*}, t_{i}\right)_{i=1, \ldots, n}$.

Step 5: (Bootstrap replication). Repeat step 1-4 $B$ times $\left(B=1000\right.$, say) which gives values $\hat{D}_{1}^{2, *}, \ldots, \hat{D}_{B}^{2, *}$.

Now we construct the empirical cumulative distribution function [ECDF], which can be taken as an approximation for the right side in (6), because Munk \& Ruymgaart (1999) have shown that the ECDF, based on $\hat{D}_{i}^{2, *}$, asymptotically approximates the distribution of $\hat{D}^{2}$. The ECDF can be obtained by ordering the values of $\hat{D}_{1}^{2, *}, \ldots, \hat{D}_{B}^{2, *}$ increasingly and plotting them against the value $(i) / B$, where $(i)$ denotes the position of $\hat{D}_{i}^{2, *}$ in the ordered sample $\hat{D}_{(1)}^{2, *}, \ldots, \hat{D}_{(B)}^{2, *}$. The so-called estimated evidence of the model $U$ can now be obtained by determining the position of the original statistic $\hat{D}^{2}$ in the ordered sample $\hat{D}_{(1)}^{2, *}, \ldots, \hat{D}_{(B)}^{2, *}$. This is some number $k^{*} \in\{0, \ldots, B+1\}$. From this number one computes

$\alpha^{*}=1-k^{*} / B$.

Statisticians denote $\alpha^{*}$ as the $p$-value of the test statistic $\hat{D}^{2}$. The interpretation of this value is as follows. A small $\alpha^{*}$ indicates that the observed data are very unlikely to have been generated by model $U$, because the probability that the observed (or a larger value) $\hat{D}^{2}$ occurs is very small, namely $\alpha^{*}$ (recall that the bootstrap algorithm reproduces the true distribution of $\hat{D}^{2}$ in (6)). On the other hand, if $\alpha^{*}$ is large (and hence $\hat{D}^{2}$ small) there should be rare evidence against a proper use of model $U$, because $\hat{D}^{2}$ provides a good fit of the data to the model compared to all other possible outcomes which could have occured.

A formal test at significance level $\alpha$ can be performed when deciding against $U$ if

$\alpha^{*}<\alpha$.

In other words a small $\alpha^{*}$ indicates that the deviation from the model $U$ is not simply due to noise, but rather a systematic devation from the model $U$ has to be taken into account.

We would like to close this section by making some remarks about the applicability of bootstrap algorithms in the context of goodness of fit, and giving some arguments why our bootstrap algorithm is valid in the heteroscedastic case. Stute et al. (1998) have shown that the wild bootstrap is valid in heteroskedastic models with random grid points $t$. This result can be extended to deterministic grid points, as is the case in our example, provided the scheme is not "too" wiggly (a precise formulation can be found in Munk 1999), which holds true for the subsequent example. We mention that an explanation for the wild bootstrap validity is its automatic adaptivity to inhomogeneous variances, because it can be shown that the variance in the artifical datapoints $y_{i}^{*}$ induced by "wild" resampling (step 2 in our algorithm) yields

$$
V^{*}\left[\varepsilon_{i}^{*}\right]=\hat{\varepsilon}_{i}^{2}
$$

which estimates approximately $\sigma^{2}\left(t_{i}\right)$. In contrast, the $n$ out of $n$ bootstrap (cf. Stute et al. 1998) and the residual bootstrap here fail to hold because the bootstrap variance is in the latter case $\frac{1}{n} \sum_{i=1}^{n} \hat{\varepsilon}_{i}^{2}$, which approximates the average overall variance $\iint \sigma^{2}\left(t_{1}, t_{2}\right) \mathrm{d} t_{1} \mathrm{~d} t_{2}$ in our example. This argument transfers essentially to any random quadratic form (such as $\hat{D}^{2}$ or Bi \& Börner's $1994 \chi^{2}$ statistic). The residual bootstrap is consistent only if the error is homoscedastic, which, however, in the subsequent example is not the case. The case when the model space $U$ is of dimension $\infty$, as considered by Bi \& Börner (1994), is in principle similar; here it is also well known that the residual bootstrap is insufficient in heteroscedastic models (Härdle \& Marron 1991).

\section{Application: Recovering the luminosity distribution in the Milky Way}

The DIRBE experiment on board the COBE satellite, launched in 1989, made measurements of the surface brightness in several infrared wavebands (Weiland 1994). A difficulty with the COBE/DIRBE data is that it has to be corrected against certain effects. The most important correction is the removal of dust absorption. This has been done by Spergel et al. (1996). We use their corrected COBE/DIRBE $L$-band data in our fits. The resolution of the data are $n \times m=120 \times 40$ points in $l, b$ respectively, covering a range $-89.25^{\circ} \leq l \leq 89.25^{\circ}$ and $-29.25^{\circ} \leq b \leq 29.25^{\circ}$. The points in this two-dimensional grid are equally spaced.

The COBE/DIRBE data have been used to deproject the three-dimensional density of the MW in a number of projects. A main difficulty in recovering the three-dimensional luminosity distribution from the twodimensional surface brightness distribution of the MW is that it is not a unique operation, in general. One way to avoid this problem is to fit a parametric model to the MW in order to reduce the set of possible models. Several parametric models have been suggested, see for example Kent et al. (1991), Dwek et al. (1995) or Freudenreich (1998). Another approach is to use the non-parametric Richardson-Lucy algorithm for the deprojection of the data (Binney \& Gerhard 1996; Binney et al. 1997; 
Bissantz et al. 1997), in order to reconstruct the luminosity distribution of the MW.

In parametric models of the MW density, about ten "structural parameters" - including normalisations, scale lengths and geometrical shape parameters of the bulge/bar - are used (see, for example, Kent et al. 1991; Dwek et al. 1995; Freudenreich 1998). In what follows, we assume that these parameters are selected such that the projection of a model onto the sky is an injective operation.

\subsection{The basic equations of the astrophysical problem}

We will first derive a general mathematical model of the problem of recovering the MW luminosity distribution from the $L$-band data. The projection of a threedimensional light distribution to a surface brightness (on the sky) is defined as follows. Let $\aleph$ be the set of possible luminosity densities of the MW, i.e. of maps

$\rho: \mathbb{R}^{3} \rightarrow \mathbb{R}_{\geq 0}, \quad \boldsymbol{x} \mapsto \rho(\boldsymbol{x})$

and $\Omega$ be the surface brightness distributions

$\omega:[0,2 \pi] \times\left[-\frac{\pi}{2}, \frac{\pi}{2}\right] \rightarrow \mathbb{R}_{\geq 0},(l, b) \mapsto \omega(l, b)$,

where $\omega(l, b)$ is the surface brightness at sky position $(l, b)$, and $\omega \in \Omega$. The transformation between a luminosity density $\rho$ to its corresponding surface brightness distribution is described by a linear integral operator $\mathcal{P}$. We will call this operator $\mathcal{P}$ the projection operator, since it "projects" a luminosity density on the sky, i.e. onto a surface brightness distribution.

$\mathcal{P}: \aleph \rightarrow \Omega, \quad \rho \mapsto \mathcal{P}(\rho)$

$\mathcal{P}$ is defined by the integral $\int \rho(\boldsymbol{x}) \mathrm{d} r$ of the density $\rho(\boldsymbol{x})$ along the line-of-sight from the observer to infinity in direction $(l, b)$. Let $r$ denote the distance from the observer. Note that the integrand is $\rho$ and not $\rho \times r^{2}$ because the physical extend of the observed cone $\delta \Omega$ increases as $r^{2}$ whereas the intensity of a source decreases as $r^{-2}$ and the $r$-powers therefore cancel out. Let $s(r, l, b)$ be the path from the observer to infinity along the line-of-sight to $(l, b)$, parametrized by the distance from the observer $r$. Then

$\omega(l, b)=\mathcal{P}(\rho)(l, b)=\int_{0}^{\infty} \rho(s(r, l, b)) \mathrm{d} r$.

So far we have used a spherical coordinate system centered at the observer. Coordinate axes are the sky longitude $l$ and latitude $b$ and the distance from the observer $r$. We now introduce a second coordinate system. Let $(x, y, z)$ be the coordinate axes of a cartesian, galactocentric, coordinate system, s.t. $x$ and $y$ lie in the main plane of the MW. We define $x$ to be along the major axis of the bulge/bar (cf. Sect. 5.2 for further explanation of the components of our parametric MW model, including the bulge/bar), $y$ along the minor axis, and $z$ perpendicular to the main plane of the MW. Let us further call "bar angle $\Phi$ " the angle between the major axis of the bar/bulge and the line-of-sight

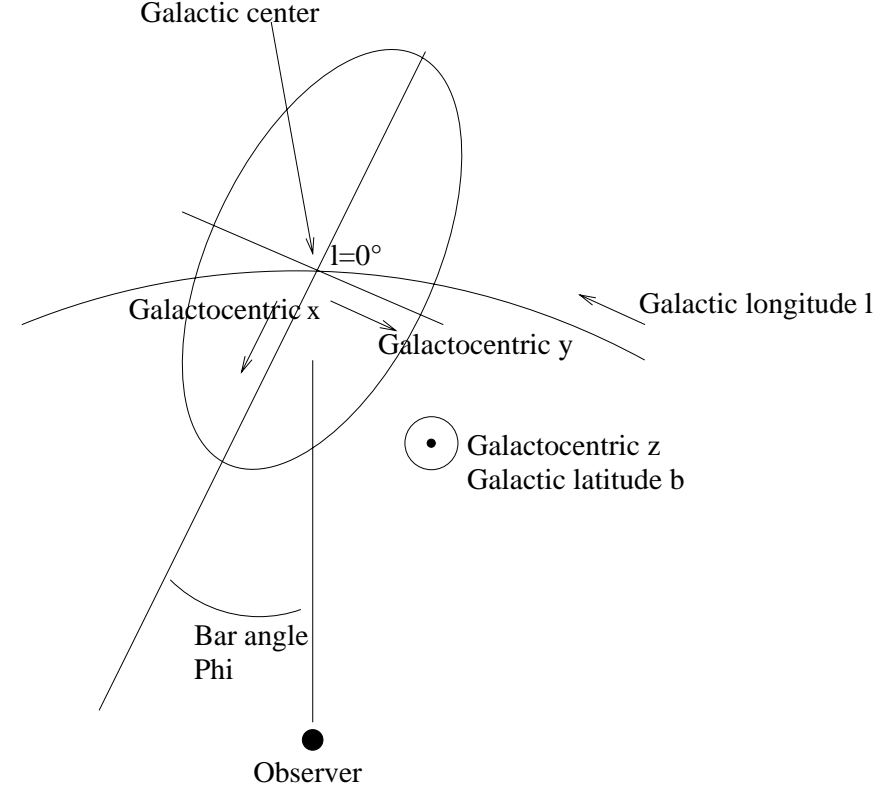

Fig. 2. A sketch of the two coordinate systems that we use in this paper. Luminosity densities of the MW are defined in the galactocentric coordinate system $x, y, z$. Galactic longitude $l$ and latitude $b$ define a position on the sky. Together with the distance from the observer $r$ they constitute the observer centered coordinate system.

direction from the observer to the galactic centre. The position of the observer in this coordinate system is denoted as $\left(x_{\odot}, y_{\odot}, z_{\odot}\right)$. Figure 2 depicts the two coordinate systems.

In our setting, a parametric model of the MW is a class $U$ of distributions $\rho_{\vartheta}$ such that

$U=\left\{\rho_{\vartheta}(\cdot)\right\}_{\vartheta \in \Theta}$

where $\Theta$ denotes a set of parameters $\Theta \subseteq \mathbb{R}^{d}$. This definition is in accordance with our terminology at the beginning of this paper. We cannot observe $\omega$ directly, due to measurement errors, dust removal from the raw data and other sources of noise. Hence, our observations $y_{i j} \equiv \omega\left(l_{i}, b_{j}\right)+\varepsilon_{i j}$ are blurred by some random error $\varepsilon_{i j}$, the distribution of which may vary between different sky positions $\left(l_{i}, b_{j}\right)$. Particularly, we will see in the following that it is necessary to allow for a position-dependent noise $\operatorname{Var}\left[\varepsilon_{i, j}\right]=\sigma_{i, j}^{2}$. Therefore our astrophysical problem is to reconstruct $\rho$ from the noisy integral equation.

$y_{i j}=\mathcal{P}(\rho)\left(l_{i}, b_{j}\right)+\varepsilon_{i j}$.

Note that this is a noisy Fredholm equation of the first kind as in (4); the suggested method in the last section transfers directly to the present setting. Note, that $\mathcal{P}$ is a linear injective operator as long as $\rho>0$ due to our selection of the parametric model.

Let

$\omega_{\vartheta}(l, b)=\mathcal{P}\left(\rho_{\vartheta}\right)(l, b) ; \quad \vartheta \in \Theta$,

and consider the transformed model

$V_{\mathbf{T}}=\mathbf{T} V=\left\{\mathbf{T} \omega_{\vartheta}(l, b)\right\}_{\vartheta \in \Theta}$, 
where

$V=\mathcal{P} U=\left\{\omega_{\vartheta}(l, b)\right\}_{\vartheta \in \Theta}$

and

$\mathbf{T}[\omega]\left(l^{\prime}, b^{\prime}\right)=\iint \omega(l, b) T\left(\left(l^{\prime}, b^{\prime}\right),(l, b)\right) \mathrm{d} l \mathrm{~d} b$.

Specific models $U$ will be discussed in the next section Following the approach in Sect. 4 we specify the smoothing integral operator $\mathbf{T}$ by defining the smoothing kernel as

$T\left(\left(l^{\prime}, b^{\prime}\right),(l, b)\right)=\min \left\{l, l^{\prime}\right\} \cdot \min \left\{b, b^{\prime}\right\} ;(l, b),\left(l^{\prime}, b^{\prime}\right) \in \mathbb{R}^{2}$.

This amounts to a kind of cumulative smoothing, which downweights small-scale features in the data, and emphasizes trends on large scales.

Now, as a first step, we estimate $g\left(l^{\prime}, b^{\prime}\right)=\mathbf{T}[\omega]\left(l^{\prime}, b^{\prime}\right)$ by

$\hat{g}\left(l^{\prime}, b^{\prime}\right)=\frac{1}{n \cdot m} \sum_{i=1}^{n} \sum_{j=1}^{m} y_{i j} T\left(\left(l^{\prime}, b^{\prime}\right),\left(l_{i}, b_{j}\right)\right)$

and determine numerically the "transformed" LSE

$\hat{\vartheta}_{\mathbf{T}}=\operatorname{argmin}_{\vartheta \in \Theta}\left\|\hat{g}-\mathbf{T}\left[\omega_{\vartheta}\right]\right\|^{2}$

where $\|\cdot\|^{2}$ denotes the usual $L^{2}$-norm. Finally, the minimising value

$\hat{D}^{2}=\left\|\hat{g}-\mathbf{T}\left[\mathcal{P}\left(\rho_{\hat{\vartheta}_{\mathbf{T}}}\right)\right]\right\|^{2}$

is computed. Now the bootstrap algorithm in Sect. 4 can be applied. Finally, we mention that for the minimization in (8) we have used the Marquardt-Levenberg algorithm (Press et al. 1994).

\subsection{A parametric model of the Milky Way}

We will now investigate whether the functional form of the luminosity distribution of the MW as suggested by BGS provides a satisfactory fit to the COBE/DIRBE $L$-band data. This functional form is a superposition of a doubleexponential disk with a truncated power-law bulge

$$
\begin{aligned}
& \rho_{\vartheta}(x, y, z)= \underbrace{d \cdot\left(\frac{\mathrm{e}^{-|z| / z_{0}}}{z_{0}}+\alpha \cdot \frac{\mathrm{e}^{-|z| / z_{1}}}{z_{1}}\right) \cdot r_{\mathrm{d}} \cdot \mathrm{e}^{-r / r_{\mathrm{d}}}}_{\text {disk }} \\
&+\underbrace{b \cdot \frac{\mathrm{e}^{-a^{2} / a_{\mathrm{m}}^{2}}}{a_{\mathrm{m}}^{3} \cdot \eta \cdot \zeta \cdot\left(1+a / a_{\mathrm{c}}\right)^{q}}}_{\text {bulge/bar with cusp }} \\
& a^{2} \equiv x^{2}+\left(\frac{y}{\eta}\right)^{2}+\left(\frac{z}{\zeta}\right)^{2} \\
& r^{2} \equiv x^{2}+y^{2},
\end{aligned}
$$

where the parameter $\vartheta$ can be devided into "structural" parameters $\left(z_{0}, z_{1}, r_{\mathrm{d}}, b, a_{\mathrm{m}}, \eta, \zeta, a_{\mathrm{c}}, d, q\right)$ that specify the functional form of the model and "geometrical" parameters that define the position of the sun in the coordinate system. The "geometrical" parameters are fixed in advance and are the position of the sun above the main plane of the $\mathrm{MW}, z_{\odot}=14 \mathrm{pc}$, the distance of the sun from the galactic centre projected on the main plane, $r_{\odot}=8 \mathrm{kpc}$ and the bar angle, $\phi=20^{\circ}$ (BGS). It is not feasible to estimate the cusp parameters $a_{\mathrm{c}}=100 \mathrm{pc}$ and $q=1.8$ from our data, because the available resolution is not sufficient. We use the same values as BGS.

As a first step we will investigate graphically whether an inhomogeneous variance pattern has to be assumed, which is indicated by inhomogeneous squares of residuals. Figure 3 shows the COBE/DIRBE $L$-band data and Fig. 4 the residuals $r_{i j}^{2}=\left(y_{i j}-\omega_{\hat{\vartheta}_{\mathrm{BGS}}}\right)^{2}$, with the model parameters $\hat{\vartheta}_{\text {BGS }}$ taken from BGS. Provided this model holds (approximately) true, as an important conclusion from Fig. 4 we find strong indication for inhomogeneous noise. Interestingly, towards the boundary of the observed part of the sky, the variability of the observations increases. Figure 6 shows the difference between the model and the data including the algebraic sign of the difference. Note that the error distribution is obviously inhomogeneous, both in the logarithmic magnitude scale plotted in the figures and in a linear scale. Further note there is a systematic dependence of the sign of the deviations on the position on the sky, whereas the model fits well in the central part of the observed part of the sky. This is indication that the MW disk shows deviations from an exponential $z$-dependence.

We now determine the best-fit model parameter $\hat{\theta}^{*}$ by minimisation of $\hat{D}^{2}$. We use the parameters found by BGS as starting values for our minimisation algorithm. Due to the increasing noise towards the boundary of the observed part of the sky, we restrict the region of the surface brightness data used in the fit to the region $|l| \leq 60^{\circ}$ and $-20^{\circ} \leq b \leq 10^{\circ}$. This is done to downweight those parts of the sky where noninformative parts in the data are expected (see Fig. 4). Figure 5 shows this data after it has been smoothed with the smoothing operator T. Note how much smoother the smoothed data appears compared to the original COBE/DIRBE $L$-band data. Our computational strategy consists of two steps.

1. Fitting the disk: in the first step we fit the disk parameters with fixed bulge parameters;

2. Fitting the bulge/bar: in the second step we fix the disk related parameters found in the first step (except for the normalisation parameter $d$ ) and fit the bulge/bar parameters and $d$.

Table 1 shows our result for the best-fit model parameters $\hat{\vartheta}^{*}$ and the model parameters of BGS. As suggested in Sect. 4 we have obtained our best-fit model parameters by minimisation of $\hat{D}^{2}$. Note that BGS have not used exactly the same region of the sky in their fit as we use here. Therefore, one has to take into account that differences between these model parameters may be partially due to different regions of the sky (data) used in the fit. We reduce this problem by redetermining the 


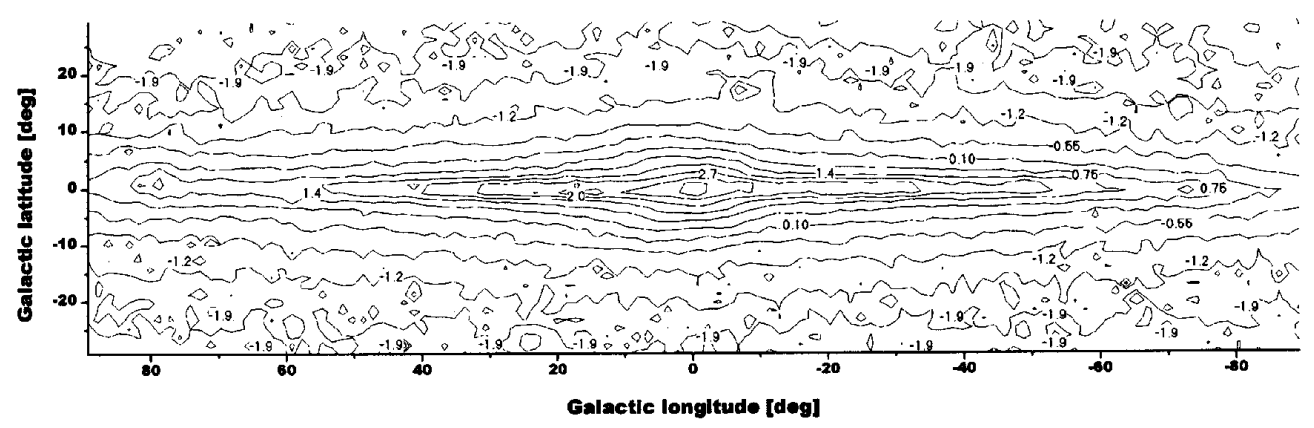

Fig. 3. COBE/DIRBE $L$-band data. Contours levels are given in magnitudes. Note that contour levels are only defined up to a common offset.

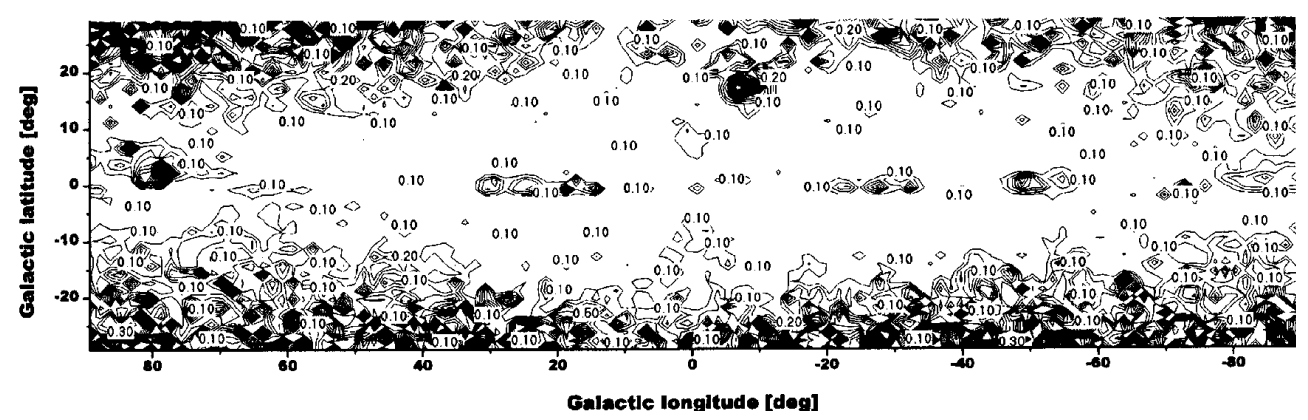

Fig. 4. Square difference between COBE/DIRBE $L$-band data logarithmic surface brightness (magnitudes) and the parametric model with the parameters from BGS. Contours levels are $n \times 0.1 \mathrm{mag}^{2}, n \in \mathbb{N}$.

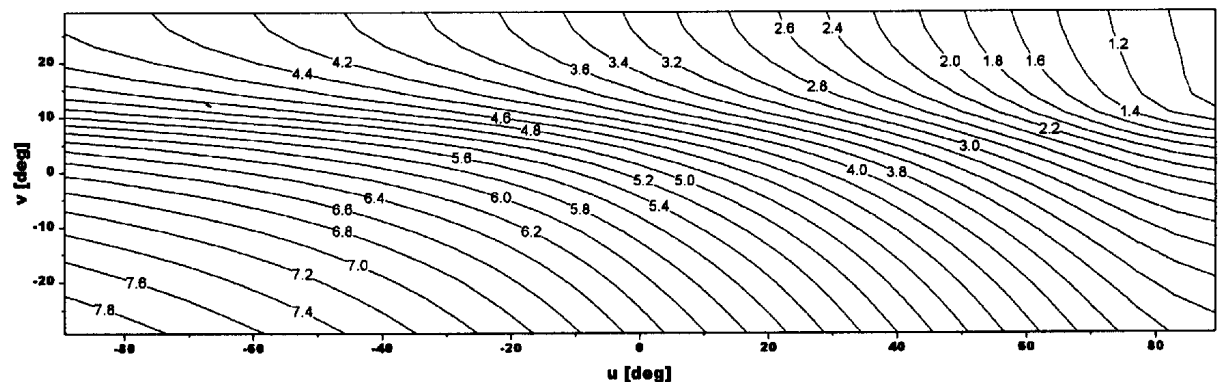

Fig. 5. The smoothed COBE/DIRBE $L$-band data. This is the observed data in the sky region $|l| \leq 60^{\circ}$ and $-20^{\circ} \leq b \leq 10^{\circ}$, after application of the smoothing operator $\mathbf{T}$. Contours indicated are for the natural logarithm of $\mathbf{T} \omega$. Note that the $x$ and $y$ axis are from $|l| \leq 60^{\circ}$ and $-20^{\circ} \leq b \leq 10^{\circ}$, due to the definition of $\mathbf{T}$.

Table 1. Parameter values for our model and the model according to BGS and the statistical parameter $\alpha^{*}(7)$. Note that the parameters $\alpha$ and $z_{1}$ are not fitted.

\begin{tabular}{lcccccccccc}
\hline Parameters & $z_{0}$ & $z_{1}$ & $\alpha$ & $r_{\mathrm{d}}$ & $d$ & $\eta$ & $\zeta$ & $a_{\mathrm{m}}$ & $b$ & $\alpha^{*}$ \\
\hline "Our" & 0.162 & 0.042 & 0.27 & 2.56 & 0.41 & 0.502 & 0.59 & 1.90 & 306.1 & 0.86 \\
"BGS" & 0.21 & 0.042 & 0.27 & 2.5 & 0.463 & 0.5 & 0.6 & 1.9 & 234.4 & 0.80 \\
\hline
\end{tabular}

normalisations $b, d$ of the model by BGS (keeping fixed their other parameters) with our algorithm, using the region of the sky selected above. The value of our proposed statistical quantity $\alpha^{*}$ for the BGS model has been calculated for this modified version of their model parameters.

Applying the bootstrap algorithm presented in Sect. 4 to our model we find that $\alpha^{*}=0.86$, which indicates no significant evidence against this model. For the parameters found in BGS a value $\alpha^{*}=0.80$ is obtained which yields a slightly worse fit. Note that, at a first glance, this statement is in contradiction to the argument given by
BGS in the last paragraph of their p. 366. They pointed out that a graphical inspection of residuals suggests that for the model considered, some local regions of the sky seem to show systematic differences between their model and the observed data. As a conclusion we find that the proposed method is not capable of concluding that these local deviations between model and data are due to systematic deviations. As pointed out by the referee this might be due to lack of power of the proposed method, because an additional smoothing step was proposed. Indeed, this corresponds to some theoretical results concerning the 


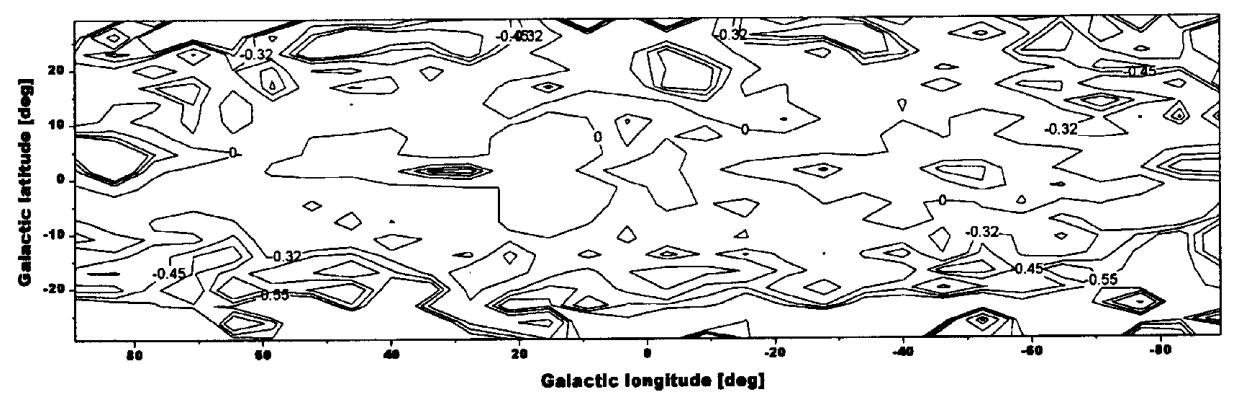

Fig. 6. Difference between COBE/DIRBE $L$-band data logarithmic surface brightness (magnitudes) and the parametric model from BGS in magnitudes. Negatives values for the contour levels indicate that a model is too bright as compared to the data. The contour levels are chosen such that their squares are equivalent to $\approx 0.1 \mathrm{mag}^{2}, \approx 0.2 \mathrm{mag}^{2}$ and $\approx 0.3 \mathrm{mag}^{2}$, to allow a direct comparison with Fig. 4.

asymptotic efficiency of the proposed method (Munk \& Ruymgaart 1999). In fact, a more powerful method could result from chosing a data-driven smoothing operator $\mathbf{T}$, similar to bandwidth selection in kernel regression. The main difficulty which arises is a different limit law compared to the case discussed in the present, where $\mathbf{T}$ is fixed. However, this is beyond the scope of this paper and will be an interesting topic for further research.

It can be seen from Table 1 that the main difference between the two models is that our model has a lower disk scale height $z_{0}$. The value $\alpha^{*}$ was found to be slightly better for our new model compared to the BGS parameters. However, recall that we used only a part of the COBE/DIRBE $L$-band surface brightness data in our fit.

\section{Results and conclusions}

We have argued that classical measures of goodness of fit adopted from checking distributional assumptions can be misleading in the context of (inverse) regression. Particularly, an inhomogeneous noise field can inflate the precision of common $\chi^{2}$ quantities. For this case, a new method was proposed for noisy Fredholm equations of the first kind by Munk \& Ruymgaart (1999). As an example for the application of the suggested algorithm, we use the problem of determining the luminosity density in the MW from surface brightness data. From this we have found that the parametric model in Binney et al. (1997) can be improved slightly and gives a satisfactory fit of the COBE/DIRBE $L$-band data in a range of $-20^{\circ} \leq b \leq 10^{\circ}$.

Acknowledgements. The authors are indebted to the organizers F. Ruymgaart, W. Stute and Y. Vardi of the conference "Statistics for Inverse Problems" held at the Mathematical Research Center at Oberwolfach, Germany, 1999. The present paper was essentially initiated by this meeting. We would like to thank O. Gerhard and the referee, P. Saha, for many helpful comments. N. Bissantz acknowledges support by the Swiss Science Foundation under grant number 20-56888.99.

\section{Appendix A: Chosing the smoothing kernel T}

We mention that our procedure can also be performed with any other smoothing kernel $T$. This will also yield in general different values of $\alpha^{*}$. In principle, a valid option is any injective Operator T. A good choice of $\mathbf{T}$, however, is driven by various aspects, such as efficiency or simplicity. An extensive simulation study performed in Munk \& Ruymgaart (1999), reveals the kernel $T(u, v)=\min (u, v)$ as a reasonable choice which yields a procedure capable to detect a broad range of deviations from $U$. See, however, the discussion in Sect. 5. A particularly simple choice in noisy inverse models

$y_{i}=\mathbf{K} f\left(t_{i}\right)+\varepsilon_{i}$

can be achieved if $\mathbf{T}$ is the adjoint of $\mathbf{K}$ itself, provided $\mathbf{K}$ is a smoothing operator of the type

$\mathbf{K} f(\cdot)=\int K(\cdot, v) f(v) \mathrm{d} v$.

However, in our application this is not easy to calculate and will depend on constraints which force the particular model $\rho_{\vartheta}$ to be identifiable.

\section{References}

Aerts, M., Claeskens, G., \& Hart, J. 1999, J. Amer. Statist. Assoc., 94, 869

Akaike, H. 1974, IEEE Trans. Autom. Control, 19, 716

Alcock, C., Allsman, R. A., Alves, D., et al. 1997, ApJ, 486, 697

Azzalini, A., \& Bowman, A. W. 1993, Jour. Roy. Statist. Soc. Ser. B, 55, 549

Babu, G. J. 1984, Shankya A, 46, 85

Barrow, J. D., Sonoda, D. H., \& Bhavsar, S. P. 1984, MNRAS, 210, 19

van den Bergh, S., \& Morbey, C. L. 1984, ApJ, 283, 598

Bertero, M. 1989, Adv. Electron. El. Phys., 75, 1

Bi, H., \& Boerner, G. 1994, A\&AS, 108, 409

Binney, J., \& Gerhard, O. 1996, MNRAS, 279, 1005

Binney, J., Gerhard, O., \& Spergel, D. 1997, MNRAS, 288, 365 (BGS)

Bissantz, N., Englmaier, P., Binney, J., et al. 1997, MNRAS, 289,651

Bowman, A. W., \& Azzalini, A. 1997, Applied smoothing techniques for data analysis: the kernel approach with S-Plus illustrations, Oxford Statistical Science Series (Oxford: Oxford University Press), 18 
Cook, R. D., \& Weisberg, S. 1999, Applied Regression Including Computing and Graphics (New York, NY: Wiley)

Cox, D. R., \& Hinkley, D. V. 1974, Problems and solutions in theoretical statistics (A Halsted Press Book, London: Chapman and Hall; New York: John Wiley \& Sons)

Cox, D., Koh, E., Wahba, G., \& Yandell, B. S. 1988, Ann. Statist., 18, 113

Davison, A. C., \& Hinkley, D. V. 1997, Bootstrap methods and their application, Cambridge Series on Statistical and Probabilistic Mathematics (Cambridge: Cambridge University Press)

Dette, H., \& Munk, A. 1998, Ann. Stat., 26, 778

Dwek, E., Arendt, R. G., Hauser, M. G., et al. 1995, ApJ, 445, 716

Efron, B. 1979, Ann. Stat., 7, 1

Efron, B., \& Tibshirani, R. J. 1993, An Introduction to the Bootstrap, Monographs on Statistics and Applied Probability (New York, NY: Chapman \& Hall), 57

Eubank, R. L., \& Hart, J. D. 1992, Ann. Stat., 20, 1412

Freudenreich, H. T. 1998, ApJ, 492, 495

Gallant, A. R. 1987, Nonlinear Statistical Models, Wiley Series in Prob. \& Math. Stat. (Wiley: New York)

Härdle, W., \& Mammen, E. 1993, Ann. Stat., 21, 1926

Härdle, W., \& Marron, J. S. 1991, Ann. Stat., 19, 778

Hart, J. D. 1997, Nonparametric smoothing and lack of fit tests, Springer Series in Statistics (Springer)

Hocking, R. R. 1996, Methods and Applications of Linear Models (New York, NY: Wiley)

Kent, S. M., Dame, T. M., \& Fazio, G. 1991, ApJ, 378, 131

Lucy, L. B. 1994a, A\&A, 289, 983

Lucy, L. B. 1994b, Rev. Mod. Astron., 7, 31
Mair, B. A., \& Ruymgaart, F. H. 1997, SIAM J. Appl. Math., 56,1424

Matthai, A. M., \& Provost, S. B. 1992, Quadratic forms in Random Variables (Marcel Dekker, NY)

Mohdeb, Z., \& Mokkadem, A. 1998, C. R. Acad. Sci., Paris, Ser. I, Math., 326(9), 1141

Müller, H. G. 1992, Scand. J. Stat., 19, 157

Munk, A. 1999, under revision, Scand. J. Stat.

Munk, A., \& Ruymgaart, F. 1999, submitted

Nychka, D. W., \& Cox, D. D. 1989, Ann. Stat., 17, 556

Press, W. H., Teukolsky, S. A., Vetterling, W. T., et al. 1994, Numerical recipes in C, 2nd Ed. (Cambridge: Cambridge University Press)

van Rooij, \& Ruymgaart, F. H. 1996, J. Stat. Plann. Infer., 53, 389

Shao, J., \& Tu, D. 1995, The Jacknife and Bootstrap (Springer, NY)

Shorack, G., \& Wellner, J. 1986, Empirical Process with Applications to Statistics (Wiley, NY)

Simpson, G., \& Mayer-Hasselwander, H. 1986, A\&A, 162, 340

Spergel, D. N., Malhotra, S., \& Blitz, L. 1995, Towards a ThreeDimensional Model of the Galaxy, in ESO/MPA Workshop on Spiral Galaxies in the Near-I, ed. D. Minniti, \& H.-W. Rix (Springer), 1996

Stute, W., Gonzáles-Manteiga, W., \& Presedo Quindimil, M. 1998, J. Amer. Stat. Assoc., 93, 141

Wand, M. P., \& Jones, M. C. 1995, Kernel smoothing, Monographs on Statistics and Applied Probability (London: Chapman \& Hall), 60

Weiland, J. L., et al. 1994, ApJ, 425, L81

Wu, C. F. J. 1986, Ann. Stat., 14, 1261 